\title{
Acceptance of correct visual acuity prescription after counseling in patients with Anisometropia
}

\begin{abstract}
Objective: This study was design to know the acceptance of anisometropic correction, degree and type of anisometropia (greater than 2.5 diopters) in children 5-15 years of age.

Material \& methods: This was an observational cross-sectional study carried out at Pediatrics Ophthalmology Department of Al-Ibrahim Eye Hospital, Karachi. The duration of study was June to November, 2017. Non-probability purposive sampling technique was used for selection of patients. Inclusion criteria were patients diagnosed with anisometropia greater than 2.5 diopters, age group 5-15 years and both sex. Maximum anisometropic correction was given to the patients according to which proforma was filled and patient was asked to come on follow up. Counseling was done for wearing prescription then follow up was conducted after four weeks or minimum of after 20 days in some patients. After then receiving patient on follow up a series of questions was asked to check the acceptance of wearing maximum anisometropic correction.
\end{abstract}

Results: A total of 53 patients were examined with anisometropia. There were 29 (54.7\%) male whereas $24(45.2 \%)$ were female. After last follow-up Anisometropic correction was accepted by $38(72 \%)$ respondents and 8 patients $(15 \%)$ lost their follow-up. However, remaining $7(13 \%)$ patients were not accepted their full anisometropic correction. Visual acuity was seen to be better after correction accepted by the respondents with P-value of 0.018 .

Conclusion: The importance of screening of children 11-15 years for refractive error and anisometropia and the importance of a future prospective study on the magnitude, cause and treatment of anisometropia at more treatable age is indicated. There were $72 \%$ patients who fully accepted their anisometropic correction as they were all first time wearer. Out of which, majority were of having a difference of 3-4 diopters.

Keywords: anisometropia, refractive error, counseling, visual acuity
Volume 7 Issue 4 - 2018

\author{
Neelam Nausheen,' Muhammad Faisal \\ Fahim, ${ }^{2}$ Paras Azam, ${ }^{3}$ Tehseen Javaid ${ }^{4}$ \\ 'Department of Ophthalmology,Al-lbrahim Eye Hospital, \\ Pakistan \\ ${ }^{2}$ Department of Biostatistics \& Research, Bahria University \\ Medical \& Dental College, Pakistan \\ ${ }^{3}$ Department of Ophthalmology,Akhter Eye Hospital, Pakistan \\ ${ }^{4}$ Department of Ophthalmology, Hashmani's Hospital, Pakistan
}

Correspondence: Muhammad Faisal Fahim, M.Sc. (Statistics), Researcher, Department of Biostatistics \& Research, Bahria University Medical \& Dental College, Karachi, Pakistan, Email faisalfahim88@hotmail.com

Received: July 04, 2018 | Published: August 20, 2018

\section{Introduction}

Anisometropia is the condition in which the two eyes have unequal refractive power, one eye may be markedly stronger than the other and it is significant cause of amblyopia. The anomaly is found in every possible variety; one eye may be emmetropic and its fellow eye of any other denomination, hypermetropic, myopic or astigmatic; or both eyes may be ametropic. ${ }^{1}$ A difference in power of two diopters or more is the accepted threshold to label the condition anisometropia. In certain types of anisometropia, the visual cortex of the brain will not use both eyes together (binocular vision), and will instead suppress the central vision of one of the eyes. If this occurs often enough during the first 10 years of life while the visual cortex is developing, it can result in lazy eye (amblyopia), a condition where even when correcting the refractive error properly, the person is still not correctable to $20 / 20 .{ }^{2}$ Children who have anisometropia, a difference in refractive error between their two eyes, are known to be at risk of amblyopia. ${ }^{3}$ Furthermore, most investigators have reported that the greater the magnitude of the anisometropia, the more severe the amblyopia tends to be ${ }^{4}$ Because anisometropic amblyopia is treatable in childhood, with optical correction alone or accompanied by patching or penalization of the nonamblyopic eye, eye care professionals advocate methods for detection of anisometropia in young children. ${ }^{5}$ Amblyopia is unilateral or bilateral decrease in best corrected visual acuity by formed vision deprivation and abnormal binocular interaction for which there is no pathology of eye and visual pathway. ${ }^{6}$ Amblyopia is a developmental visual disorder characterized by abnormal form vision and binocular functions. $^{7}$

Amblyopia is a condition of reduced visual function from abnormal visual experience caused by strabismus, anisometropia, or visual form deprivation during the critical period of visual development. ${ }^{8}$ New England College of Optometry, Boston showed prevalence of anisometropia $1.27 \%$, at 5 years of age and $5.77 \%$ at 12 to 15 years of age. Prevalence of anisometropia in the myopes was $9.64 \%$ and in the hyperopes was $13.64 \%$, both significantly higher than that in the emmetropes. The prevalence of anisometropia increases between 5 and 15 years. However, anisometropia was found to accompany both myopia and hyperopia. ${ }^{9}$ Prevalence of refractive anisometropia was estimated $5.3 \%$ in Taiwan. The prevalence and severity of refractive anisometropia increased with both myopic and hyperopic refractive error. ${ }^{10}$ Prevalence of anisometropic in Saudi Arabia was $1.91 \% .{ }^{11}$ Causes of anisometropia includes inborn defects in the eye, uneven growth in both eyes, Miscalculation of intraocular lens power during the post cataract surgery, Congenital difference between both eyes. Even people who have normal vision can have up to $5 \%$ difference in the refractive power of each eye. However, those with a 5-20\% difference will experience uneven vision (anisometropia). Causes include defects in the eye at childbirth as well as uneven size of the two eyes. ${ }^{12}$ 
Anisometropia probably has an important influence on the development of squint, particularly concomitant convergent strabismus in children. The more blurred images from eye acts as a sensory obstacle to binocular vision. This images becomes suppressed, and it often found that in cases of uniocular squint deviating eye is supervened to prevent diplopia. ${ }^{13}$ Assessment of a refractive error can only really be done via the process of refraction. In clinical ophthalmology, 'refraction' is an abbreviation for 'refractive correction' and it refers to the process by which the best possible visual acuity can be obtained for a patient. ${ }^{14}$ This study was design to know the acceptance of anisometropic correction, degree and type of anisometropia (greater than 2.5 diopters) in children 5-15 years of age.

\section{Methodology}

A prior ethical approval was taken from Al-Ibrahim Eye Hospital, Karachi. Permission was taken from parents/guardian before taking the interview and examination of selected patients. This was an observational cross-sectional study carried out at Pediatrics Ophthalmology Department Of Al-Ibrahim Eye Hospital, Karachi. The duration of study was June to November, 2017. Non-probability purposive sampling technique was used for selection of patients. Sample size was drawn from Google online software www.raosoft. com by taking $95 \%$ confidence interval, $5 \%$ margin of error and for population. Anisometropia patients was very exceptional for population. The required sample size was found to be 53 patients. Inclusion criteria were patients diagnosed with anisometropia greater than 2.5 diopters, age group 5-15 years and both sex. Exclusion criteria were patients having anisometropia less than 2.50 diopters. Having isometropic refractive error, other eye pathologies and those who did not give consent. For eye examination material used was Trial frame, Trial box, Visual Acuity charts, Retinoscope, Lensometer, Auto refractor and Ophthalmoscope. Data collection procedure was after selection of patients who meet inclusion criteria Visual Acuity was measured on Snellens chart. Complete objective and subjective refraction was carried out on each patient and each eye was tested properly. Maximum anisometropic correction was given to the patients according to which Proforma were filled and patient was asked to come on follow up. Counseling was done for wearing prescription then follow up was conducted after four weeks or minimum of after 20 days in some patients. After then receiving patient on follow up a series of questions was asked to check the acceptance of wearing maximum anisometropic correction. Visual acuity betterment was notice through Snellens chart, if the patient improves one line i.e. (before6/12, after 6/9) this was called as one/single line improvement. If patient improves two lines improvement i.e. (before 6/18, after 6/9) this was considered as two line improvement in visual acuity.

Statistical analysis: Data was entered and analyzed through software Statistical Package for social sciences (SPSS) version 23.0. Qualitative variables were presented as frequency and percentages. Bar and Pie charts were also made for data representation. Fisher exact test was applied in age and gender wise anisometropic correction. Chisquare test was applied to see the significance of visual acuity before and after correction accepted by the respondents. $\mathrm{P} \leq 0.05$ considered to be statistically significant.

\section{Results}

A total of 53 patients were recruited and examined, who meet the inclusion criteria of age \&power of refractive error, and their refractive errors corrected. Gender wise distribution showed that 29 $(54.7 \%)$ were male whereas $24(45.2 \%)$ were females. The occurrence of anisometropia was found mostly in the age group of 11-15 years that is $31(58.5 \%)$ followed by $5-10$ years of $22(41.5 \%)$.

After last follow-up Anisometropic correction was accepted by 38 $(72 \%)$ respondents and 8 patients $(15 \%)$ lost their follow-up. However, remaining $7(13 \%)$ patients were not accepted their full anisometropic correction. Fisher exact test reports there is no significant difference found in the age groups regarding acceptance of anisometropia (P-value $=0.751)$. There were $16(72.7 \%)$ respondents in 5-10 years of age whereas $22(71 \%)$ in the age group of $11-15$ years if age (Table1).

Table I Anisometropic correction with age group

\begin{tabular}{llllll}
\hline $\begin{array}{l}\text { Age } \\
\text { (years) }\end{array}$ & Accepted & $\begin{array}{l}\text { Not } \\
\text { accepted }\end{array}$ & $\begin{array}{l}\text { "Follow- } \\
\text { Up Lost" }\end{array}$ & Total & P-value \\
\hline $\begin{array}{l}5-10 \\
\text { years }\end{array}$ & 16 & 2 & 4 & 22 & \\
& $72.70 \%$ & $9.10 \%$ & $18.20 \%$ & $100.00 \%$ & \\
I5-Nov & 22 & 5 & 4 & 31 & 0.751 \\
& $71.00 \%$ & $16.10 \%$ & $12.90 \%$ & $100.00 \%$ & \\
Total & 38 & 7 & 8 & 53 & \\
& $71.70 \%$ & $13.20 \%$ & $15.10 \%$ & $100.00 \%$ & \\
\hline
\end{tabular}

When gender difference was seen in terms of acceptance of anisometropia no significant difference observed ( $\mathrm{P}$-value $=0.753$ ). There were $22(75.9 \%)$ male who accepted the correction whereas $16(66.7 \%)$ female who accepted anisometropic correction (Table 2).

Table 2 Anisometropic correction Gender wise

\begin{tabular}{llllll} 
& Accepted & $\begin{array}{l}\text { Not } \\
\text { accepted }\end{array}$ & \multicolumn{2}{c}{ "Follow-Up Lost" } & P-value \\
\hline \multirow{2}{*}{ Male } & 22 & 3 & 4 & 29 & \\
& $75.90 \%$ & $10.30 \%$ & $13.80 \%$ & $100.00 \%$ & \\
\multirow{2}{*}{ Female } & 16 & 4 & 4 & 24 & 0.753 \\
& $66.70 \%$ & $16.70 \%$ & $16.70 \%$ & $100.00 \%$ & \\
Total & 38 & 7 & 8 & 53 & \\
& $71.70 \%$ & $13.20 \%$ & $15.10 \%$ & $100.00 \%$ & \\
\hline
\end{tabular}

It was seen that after correction in both eyes betterment in vision observed. In right eye before correction contains only $1(1.9 \%)$ respondents while after correction $8(15.1 \%)$ shifted in $6 / 12$. Similarly $21(39.6 \%)$ patients shifted in vision 6/6 with P-value of 0.018 . In left eye vision was better after correction $16(30.2 \%)$ patients observed in $6 / 6$ and $8(15.1 \%)$ were found in 6/9 vision with P-value of 0.018 (Table 3).

When degree of anisometropia was noticed maximum respondents $31(58.5 \%)$ were fall in the category of $>2.5-3.25$ diopters followed by $12(22.6 \%)$ in $3.5-4.0$ diopters whereas minimum patients $4(7.5 \%)$ were seen in 4.25-5.0 diopters (Figure 1). 
Table 3 Comparison of Visual Acuity (Snellens Chart) both eyes

\begin{tabular}{|c|c|c|c|c|c|c|}
\hline & \multicolumn{3}{|c|}{ Visual acuity right eye } & \multicolumn{3}{|c|}{ Visual acuity left eye } \\
\hline & Before correction & After correction & P-value & $\begin{array}{l}\text { Before } \\
\text { correction }\end{array}$ & $\begin{array}{l}\text { After } \\
\text { correction }\end{array}$ & P-value \\
\hline HM & I (I.9\%) & 0 & & I (I.9\%) & 0 & \\
\hline $\mathrm{CF}$ & 0 & 0 & & I (1.9\%) & 0 & \\
\hline $1 / 60$ & $4(7.5 \%)$ & I (I.9\%) & & I (1.9\%) & $\mathrm{I}(1.9 \%)$ & \\
\hline $2 / 60$ & $4(7.5 \%)$ & I (I.9\%) & & I (1.9\%) & 0 & \\
\hline $3 / 60$ & $4(7.5 \%)$ & I (I.9\%) & & $4(7.5 \%)$ & I (I.9\%) & \\
\hline $6 / 60$ & 7 (I3.2\%) & 0 & & $8(15.1 \%)$ & $5(9.4 \%)$ & \\
\hline $6 / 36$ & $6(11.3 \%)$ & $5(9.4 \%)$ & 0.018 & $12(22.6 \%)$ & $4(7.5 \%)$ & 0.018 \\
\hline $6 / 24$ & $3(5.7 \%)$ & 7 (13.2\%) & & $4(7.5 \%)$ & $4(7.5 \%)$ & \\
\hline $6 / 18$ & $3(5.7 \%)$ & 0 & & $6(11.3 \%)$ & $8(15.1 \%)$ & \\
\hline $6 / 12$ & I (I.9\%) & $8(15.1 \%)$ & & $3(5.7 \%)$ & $6(11.3 \%)$ & \\
\hline $6 / 9$ & $9(17.0 \%)$ & $4(7.5 \%)$ & & $2(3.8 \%)$ & $8(15.1 \%)$ & \\
\hline $6 / 6$ & II (20.8\%) & $21(39.6 \%)$ & & $10(18.9 \%)$ & $16(30.2 \%)$ & \\
\hline Total & 53 & 53 & & 53 & 53 & \\
\hline
\end{tabular}

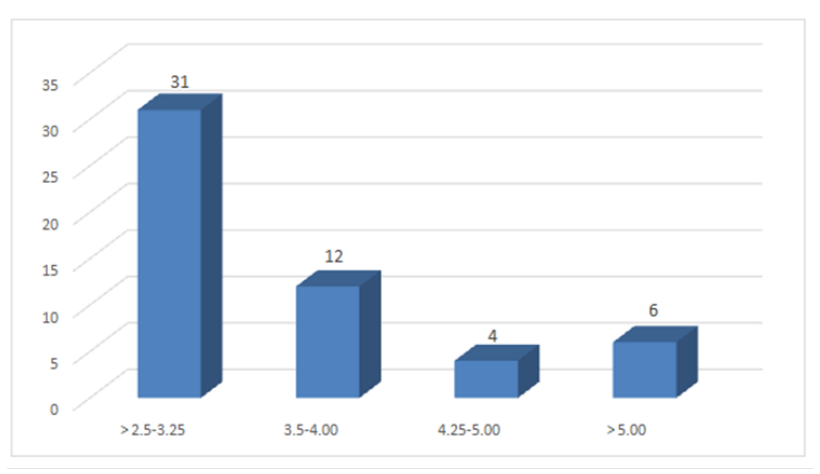

Figure I Degree of anisometropia (Diopters).

Different types of anisometropia were found in this study. Myopic anisometropia contains 25 (47.2\%) patients, Astigmatic anisometropia observed in $14(26.4 \%)$ followed by $12(22.6 \%)$ seen with hyperopic anisometropia and only 2 (3.8\%) with antimetropia (Figure 2 ).

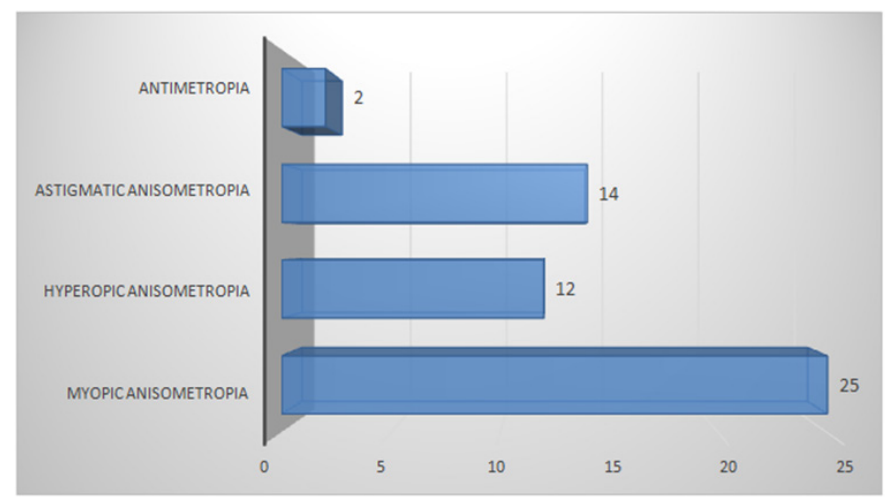

Figure 2 Type of Anisometropia.

\section{Discussion}

Anisometropia is a major cause of amblyogenic factor especially in young childrens, causing lazy eye which should be treated early in life if not treated in early 8 years of life eye become suppress for forever (amblyopia). It cannot be treated with refraction and other treatments in later life so it is very important to give the maximum anisometropic correction which could be tolerable for the patient to protect patients from later complications which include compromised vision, loss of stereopsis. ${ }^{15}$ In this study $54.7 \%$ was male and $45.3 \%$ female who are affected from anisometropia showing that males were more affected than females. There were $42 \%$ patients diagnosed with anisometropia lying in the age group of 5-10 years whereas $58 \%$ found in the age group of 11-15 years. A study reported by Cheema $\mathrm{N}$ et al presented results prevalence of anisometropia is relatively stable, individual patients develop, lose, or have changes in the magnitude of their anisometropia when followed longitudinally. ${ }^{16}$ In the current study, After final follow-up Anisometropic correction was accepted by 38 (72\%) respondents and 8 patients (15\%) lost their follow-up. However, remaining 7 (13\%) patients were not accepted their full anisometropic correction. This was a great achievement by the patients that their vision will be better in future after using the anisometropic correction at a long time continuously. It was also seen that after correction in both eyes betterment in vision observed. In right eye before correction contains only $1(1.9 \%)$ respondents while after correction $8(15.1 \%)$ shifted in 6/12. Similarly 21 (39.6\%) patients shifted in vision 6/6. In left eye vision was better after correction $16(30.2 \%)$ patients observed in $6 / 6$ and $8(15.1 \%)$ were found in $6 / 9$ vision. When degree of anisometropia was noticed maximum respondents 31 (58.5\%) were fall in the category of $>2.5-3.25$ diopters followed by $12(22.6 \%)$ in $3.5-4.0$ diopters whereas minimum patients $4(7.5 \%)$ were seen in 4.25-5.0 diopters.

In the light of the previous studies, anisometropia is one of the leading causes of amblyopia and the mechanism of anisometropic 
amblyopia is poorly understood. While it is generally agreed that anisometropic refractive errors should be corrected in patients with established amblyopia or strabismus, it is not clear what levels of anisometropia should be empirically corrected in otherwise healthy children and at what age such correction should be made to ensure optimal visual development and maturation. This question is important in preventing and managing amblyopia, because the available data supports the notion that uncorrected anisometropic response during critical periods can alter the binocular response. ${ }^{17}$ Due to this, this study was carried out to find out the maximum acceptance of anisometropia which could be tolerable for the patients.

\section{Conclusion}

The importance of screening of children 11-15 years for refractive error and anisometropia and the importance of a future prospective study on the magnitude, cause and treatment of anisometropia at more treatable age is indicated. There were $72 \%$ patients who fully accepted their anisometropic correction as they were all first time wearer. Out of which, majority were of having a difference of 3-4 diopters.

\section{Acknowledgements}

None.

\section{Conflict of interest}

Author declares that there is no conflict of interest.

\section{References}

1. Levi DM, McKee SP, Movshon JA. Visual deficits in anisometropia. Vision Research. 2011;51:48-57.

2. Astle WF, Rahmat J, Ingram AD, et al. Laser-assisted subepithelial keratectomy for anisometropic amblyopia in children:Outcomes at 1 year. Journal of Cataract \& Refractive Surgery. 2007;33(12):2028-2034.

3. Donahue SP, Arnold RW, Ruben JB, For the AAPOS Vision Screening Committee. Preschool vision screening: what should we be detecting and how should we report it?-uniform guidelines for reporting results of preschool vision screening studies. J AAPOS. 2003;7:314-316.
4. Caputo R, Frosini R, De Libero C, et al. Factors influencing severity of and recovery from anisometropic amblyopia. Strabismus. 2007;15:209-214.

5. Leon A, Donahue SP, Morrison DG, et al. The agedependent effect of anisometropia magnitude on anisometropic amblyopia severity. J AAPOS. 2008;12(2):150-156.

6. Donahue SP. The relationship between anisometropia, patient age, and the development of amblyopia. Trans Am Ophthalmol Soc. 2005;103:313336.

7. Lee CE, Lee YC, Lee SY. Factors Influencing the Prevalence of Amblyopia in Children with Anisometropia. Korean J Ophthalmol. 2010;24(4):162165 .

8. Chau B, Mitchell P. Consequences of amblyopia on education, occupation, and long term vision loss. Br J Ophthalmol. 2004;88(9):1119-1121.

9. Deng Li, Gwiazda JE. Anisometropia In Children From Infancy to 15 years. Invest Ophthalmol Vis Sci. 2012;53(7):3782-3787.

10. Hejtman JF. Prevalence and Association of Refractive Anisometropia with Near Work habits amoung Young School Children The Evidence From a Population Base Study. Public Library of science. 2012;12(1):56-59.

11. Qureshi MA, Ahmed MI, Al-debasi YH, et al. Prevalence of Incidental Amblyopia in Buraidah City, Department of Optometry. Pak J Ophthalmology. 2012;28(3):32-36.

12. https://hubpages.com/health/Anisometropia

13. Khurrana. Theory and Practice of Optics \& Refraction. India: Elsevier; 2nd Revised edition.

14. Marry Lowth. Refraction and Refractive Errors. 2016.

15. Barrett BT, Bradley A, Candy TR. The Relationship between Anisometropia and Amblyopia. Prog Retin Eye Res. 2013;36:120-158.

16. Cheema N, Anwar S, Ehsan E. Frequency of Anisometropic Amblyopia in Anisometropes Among the Age Group of 5-35 Years. APMC. 2017;11(1):55-59.

17. Dadeya S, Kamlesh, Shibal F. The effect of anisometropia on binocular visual function. Indian J Ophthalmol. 2001;49(2):261-265. 